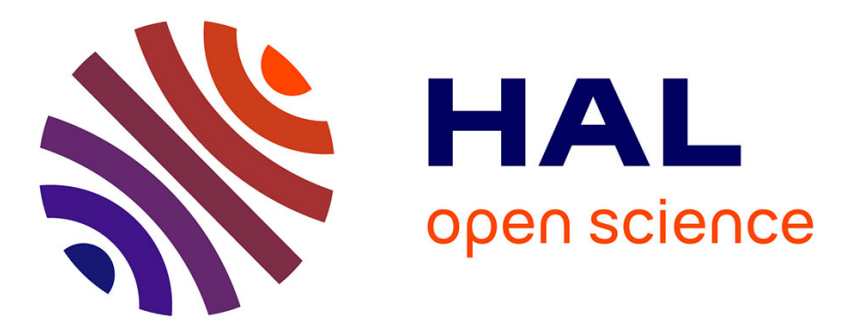

\title{
Active metamaterials with broadband controllable stiffness for tunable band gaps and non-reciprocal wave propagation
}

Kaijun Yi, Morvan Ouisse, Emeline Sadoulet, Gaël Matten

\section{- To cite this version:}

Kaijun Yi, Morvan Ouisse, Emeline Sadoulet, Gaël Matten. Active metamaterials with broadband controllable stiffness for tunable band gaps and non-reciprocal wave propagation. Smart Materials and Structures, 2019, 28 (6), pp.065025:1-13. 10.1088/1361-665X/ab19dc . hal-02160914

\section{HAL Id: hal-02160914 \\ https://hal.science/hal-02160914}

Submitted on 20 Jun 2019

HAL is a multi-disciplinary open access archive for the deposit and dissemination of scientific research documents, whether they are published or not. The documents may come from teaching and research institutions in France or abroad, or from public or private research centers.
L'archive ouverte pluridisciplinaire HAL, est destinée au dépôt et à la diffusion de documents scientifiques de niveau recherche, publiés ou non, émanant des établissements d'enseignement et de recherche français ou étrangers, des laboratoires publics ou privés.

\section{(c)(1)}

Distributed under a Creative Commons Attribution| 4.0 International License 


\title{
Active metamaterials with broadband controllable stiffness for tunable band gaps and non-reciprocal wave propagation
}

\author{
Kaijun $\mathrm{Yi}^{\mathrm{a}, *}$, Morvan Ouisse ${ }^{\mathrm{a}}$, Emeline Sadoulet-Reboul ${ }^{\mathrm{a}}$, Gaël Matten ${ }^{\mathrm{a}}$ \\ ${ }^{a}$ Univ. Bourgogne Franch-Comté, FEMTO-ST Institute, CNRS/UFC/ENSMM/UTBM, Department of \\ Applied Mechanics, 24 rue de l'épitaphe, 25000 Besançon, France
}

\begin{abstract}
One dimensional active metamaterials with broadband controllable bending stiffness are studied in this paper. The key unit of the active metamaterials is composed of a host beam and piezoelectric patches bonded on the beam surfaces. These patches serve as sensors or actuators. An appropriate feedback control law is proposed in order to change the bending stiffness of the active unit. The input of the control law is the voltage on the sensors, the output is the voltage applied on the actuators. Due to the control, bending stiffness of the active unit is $(1+\alpha)$ times of that of the bare host beam, $\alpha$ being a design parameter in the control law. The bending stiffness can be tuned to desired value by changing $\alpha$. The performances of the controlled bending stiffness are analytically and numerically studied, the stability issues are also discussed. The active units are first used in a spatial periodic waveguide to have tunable band gaps, then they are integrated in a spatiotemporal periodic waveguide to realize non-reciprocal wave propagation. Performances of the two waveguides are numerically studied.
\end{abstract}

Keywords: metamaterials, piezoelectric materials, feedback control, vibration, non-reciprocal wave propagation

\section{Introduction}

Metamaterials are artificially engineered structures with unconventional effective properties. They are composed of unit cells (also called meta-atoms [1]), whose sizes are smaller than the wavelengths at interested frequency ranges. Typically through a periodic arrangement (but not necessary [2]) of these unit cells, band gaps at low frequencies can be created for sound and vibration mitigation $[3,4]$. Since the effective properties of metamaterials can be delicately customized and tailored through the design of unit cells, metamaterials have also been widely used to control acoustic and elastic waves. A plenty of unconventional wave propagation effects have been realized using metamaterials, such as negative refraction [5], cloaking [6], topologically protected wave propagation[7], etc. Although metamaterials have helped to broaden the frontiers of acoustic and mechanical techniques in the past decades, efforts have mostly been dedicated to passive and static

\footnotetext{
${ }^{*}$ Corresponding author

Email address: kaijun.yi@femto-st.fr (Kaijun Yi)
} 
unit cells, which are difficult to be altered after being manufactured. This limitation conflicts with the demands of more intelligent and adaptive structures.

Mainly motivated by the aforementioned reason, there has been a growing effort to study active metamaterials [8-10]. The key distinguished features of these advanced metamaterials are their controllable properties. The control is mostly realized by using smart materials in the unit cell. For example, including piezoelectric patches shunted with resonant circuits into a unit cell can obtain tunable equivalent dynamic stiffness $[11,12]$. Negative capacitances have the ability to change the equivalent static Young's modulus of piezoelectric materials in a large frequency band. Therefore, they are more widely used to design controllable unit cell. The negative capacitance presents an unstable zone which must be avoided in practical applications $[13,14]$. Active control is an alternative way to tune the structural properties. Parameters of some smart materials can be tuned through an external field, for example, magnetoelastic materials show a varying Young's modulus when they are placed in a changing magnetic field. Therefore, these materials have been used to actively modulate system properties [15, 16]. Actively tuning the effective parameters using feedback control loops has also been proposed. A part of the efforts has been dedicated to design digital circuits $[17,18]$. The digital circuit measures the voltage on a piezoelectric transducer and feedbacks current into the same transducer according to a designed control law therefore to mimic the behaviors of analog electrical elements or any behavior of interest [19]. For example, the digital circuit could be programmed to mimic a negative capacitance to control the effective Young's modulus. Direct feedback control is another active way to tune the structural properties, which has already been used to realize effective negative mass [20], or add a positive active stiffness into the system [21].

Active metamaterials have been proposed for many applications. For example, periodic arrays of piezoelectric patches shunted with resonant circuits or negative capacitances are bonded on the surfaces of beams or plates to obtain tunable band gaps $[11,13,22,23]$. A self-adaptive metamaterial beam with digital circuit controlled mechanical resonators for broadband wave attenuation at sub-wavelength scales is proposed in [24]. Active metamaterials are also explored to manipulate wave propagation. Piezoelectric patches with shunts are used to steer waves for effects like wave focusing [25], wave redirecting [26]. A programmable metasurface with sensing and actuating units is proposed to manipulate the amplitude and phase of transmitted and reflected waves in real-time [27]. The metasurface particularly shows potential applications in one-way blocking of waves and cloaking. An active metamaterial consisting of symmetrical double Helmholtz resonators is proposed in [28] to realize cloak effect in fluid.

The progress of active metamaterials encourages studies on time-dependent structures. These types of structures possess parameters being modulated in time or in time and space simultaneously. It is shown that modulation of parameters of periodic waveguides in time domain significantly alters the transmission properties at frequencies near and within the band gap [29]. Piezoelectric patches shunted with time-varying resistance-inductance circuits have shown to provide broadband vibration control effect [30]. Recently, media with parameters modulated in both time and space in a traveling wave form have drawn lots of attention since the wave propagation in them is non-reciprocal. Dispersion curves of waves in these spatiotemporal periodic structures are no longer symmetrical [31]. Band 
gaps for waves propagating in opposite directions are at different frequency ranges. Within these band gaps, several unusual wave propagation behaviors have been observed, such as one-way wave transmission [31-33], frequency conversion [34] and frequency splitting [35].

Although several strategies have been proposed to design active metamaterials as introduced above, efficiently changing the structural parameters still remains an open challenge. The equivalent stiffness obtained using piezoelectric patches shunted with resonant circuits strongly depends on frequency and is only available in a narrow band near the resonant frequency of the circuit $[11,12]$. Negative capacitances are able to tune structural properties in a wide frequency band. Nevertheless, the controlled equivalent Young's modulus only varies dramatically at the vicinity of the unstable zone [13, 14], which means that to obtain significantly modulated system parameters, the system has to work very close to the unstable zone, a small variation of the applied negative capacitance value may make the system unstable or deviate the controlled parameters from the desired values. Direct active feedback control is an emerging technique to design controllable metamaterials. It has been proposed to control wave propagation and vibration properties of $1 \mathrm{D}$ periodic waveguides $[21,36]$. However, its ability to control structural parameters has not been satisfactorily explored yet.

This paper proposes new kinds of metamaterials with broadband controllable stiffness based on direct active feedback control. The designed basic active unit is composed of a host beam and piezoelectric patches bonded on the beam surfaces. Some of the patches serve as sensors to measure the input signal for the controller; the rest are used as actuators, a feedback voltage generated by the controller is applied on them. The bending stiffness of the active unit is controlled, the geometry and control law are introduced in section 2. Performance and stability issues of the designed active unit are discussed in section 3. The active units are used to form a 1D spatial periodic waveguide and a 1D spatiotemporal periodic waveguide, properties of the two waveguides are studied in section 4.1 and section 4.2, respectively. Finally, conclusions are drawn in section 5.

\section{The designed unit and control law}

Figure 1 shows the designed active unit. There are four sensors and two actuators in the cell all made of piezoelectric materials. The polarization of these patches is along the $z$ axis. Electrodes of the patches are on the surfaces perpendicular to the $z$ axis. The target is to control the bending stiffness associated with the flexural waves traveling along the $x$ axis in beam-like structures. Therefore, the four sensors are connected in a way shown in the figure to filter the voltages generated by longitudinal and zero-order torsion waves. The measured voltage by the sensors is $V_{s}$. The two actuators are also connected together, the applied voltage on them is $V_{a}$. In practice, the two sensors and one actuator on the upper surface, also those on the lower surface, may be realized using a single complete patch by dividing the electrode into three segments [37].

Under bending movement, according to the Euler-Bernoulli beam theory, the normal strain on the cross section (on YZ plane) of the active unit is

$$
\epsilon_{x}=-z \frac{\partial^{2} w}{\partial x^{2}}
$$




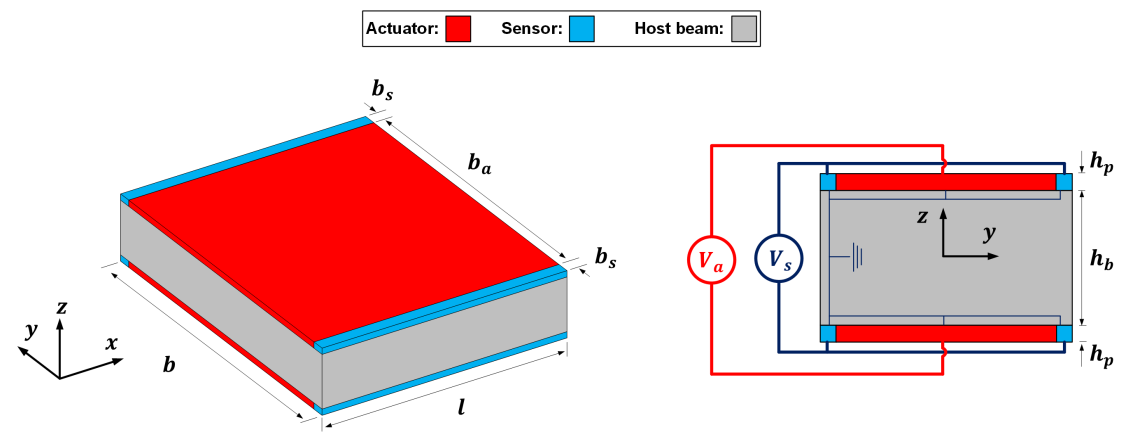

Figure 1: The designed active unit.

Hereafter, $\epsilon_{x}^{b}$ is used to represent the normal strain on the host beam cross section and $\epsilon_{x}^{p}$ is used to represent that on the patch cross section.

The constitutive equations of the piezoelectric patches are

$$
\begin{array}{r}
\epsilon_{x}^{p}=\frac{\sigma_{x}^{p}}{Y_{p}}+d_{31} E_{3}, \\
D_{3}=d_{31} \sigma_{x}^{p}+\varepsilon_{3}^{\sigma} E_{3}, \\
E_{3}=-\frac{V}{h_{p}},
\end{array}
$$

in which, $Y_{p}$ is the in-plane Young's modulus of the patch, $d_{31}$ is the piezoelectric constant under constant stress, and $\varepsilon_{3}^{\sigma}$ represents the dielectric permittivity. $\sigma_{x}^{p}$ is the normal stress on the cross section of the patch, $D_{3}$ and $E_{3}$ are the electric displacement and electric field, respectively. $V$ denotes the voltage on the electrode, for the actuator it is $V_{a}$, and for the sensor it is $V_{s}$. Directions 1, 2 and 3 correspond to $x, y$ and $z$ axis, respectively.

The normal stresses on the cross section of the actuators are obtained according to equations (2):

$$
\sigma_{x}^{p}=Y_{p}\left(\epsilon_{x}^{p}+d_{31} \frac{V_{a}}{h_{p}}\right) .
$$

The normal stress on the host beam cross section is

$$
\sigma_{x}^{b}=Y_{b} \epsilon_{x}^{b}
$$

in which, $Y_{b}$ is the Young's modulus of the beam.

According to the Euler-Bernoulli beam theory and using the expressions in equations (1), (3) and (4), the bending moment of the active unit is obtained as

$$
\begin{aligned}
M & =\int_{-\frac{h_{b}}{2}-h_{p}}^{\frac{h_{b}}{2}+h_{p}} \int_{-\frac{b}{2}}^{\frac{b}{2}} z \sigma_{x} d y d z \\
& =-\frac{Y_{b} b h_{b}^{3}}{12} \frac{\partial^{2} w}{\partial x^{2}}-\left\{\frac{Y_{p} b_{a}\left[\left(h_{b}+2 h_{p}\right)^{3}-h_{b}^{3}\right]}{12} \frac{\partial^{2} w}{\partial x^{2}}-Y_{p} d_{31} b_{a}\left(h_{b}+h_{p}\right) V_{a}\right\} .
\end{aligned}
$$

On the right side of equation (5), the first term corresponds to the contribution of the host beam, and the rest terms are contributions from the actuators. Note that, the 
contributions of the sensors are ignored since the width of them is much smaller than that of the actuators.

It is more meaningful to consider the behavior of the whole unit rather than a single section of it since the patches act on the whole unit. Therefore, equation (5) is integrated from the left end $\left(x_{L}\right)$ of the active unit to the right end $\left(x_{R}\right)$ :

$$
\int_{x_{L}}^{x_{R}} M d x=-\frac{Y_{b} b h_{b}^{3}}{12} \theta_{\triangle}-\left\{\frac{Y_{p} b_{a}\left[\left(h_{b}+2 h_{p}\right)^{3}-h_{b}^{3}\right]}{12} \theta_{\triangle}-Y_{p} d_{31} b_{a} l\left(h_{b}+h_{p}\right) V_{a}\right\},
$$

in which, $\theta_{\triangle}=\int_{x_{L}}^{x_{R}} \frac{\partial^{2} w}{\partial x^{2}} d x=\left.\frac{\partial w}{\partial x}\right|_{x_{R}}-\left.\frac{\partial w}{\partial x}\right|_{x_{L}}$, it is the difference of the rotation angles at the left and right ends.

According to equation (6), it can be seen that, the bending moment can be changed by controlling the voltage $V_{a}$. Therefore, if the voltage is controlled according to the following law

$$
V_{a}=\frac{\alpha Y_{b} b h_{b}^{3}-Y_{p} b_{a}\left[\left(h_{b}+2 h_{p}\right)^{3}-h_{b}^{3}\right]}{12 Y_{p} d_{31} b_{a} l\left(h_{b}+h_{p}\right)} \theta_{\triangle}
$$

in which, $\alpha$ is an input parameter to determine the bending stiffness of the unit after control as will be seen below, the integration of the bending moment accordingly turns into

$$
\int_{x_{L}}^{x_{R}} M d x=-(1+\alpha) \frac{Y_{b} b h_{b}^{3}}{12} \theta_{\triangle}
$$

which means that the bending stiffness of the active unit after control is

$$
D_{b}(\alpha)=(1+\alpha) \frac{Y_{b} b h_{b}^{3}}{12} .
$$

From equation (9), it can be seen that the bending stiffness of the controlled unit is $(1+\alpha)$ times of that of the bare host beam.

The sensors are open-circuited, the difference between the rotation angles $\theta_{\triangle}$ in the control law in equation (7) is measured according to

$$
\theta_{\triangle}=-\frac{2 l\left(\varepsilon_{3}^{\sigma}-Y_{p} d_{31}^{2}\right)}{h_{p}\left(h_{b}+h_{p}\right) d_{31} Y_{p}} V_{s}
$$

More details related to equation (10) can be found in Appendix B.

Using equations (7) and (10), the final control law is obtained as

$$
V_{a}=\frac{\left\{\alpha Y_{b} b h_{b}^{3}-Y_{p} b_{a}\left[\left(h_{b}+2 h_{p}\right)^{3}-h_{b}^{3}\right]\right\}\left(\varepsilon_{3}^{\sigma}-Y_{p} d_{31}^{2}\right)}{6 Y_{p}^{2} b_{a} h_{p}\left(h_{b}+h_{p}\right)^{2} d_{31}^{2}} V_{s}
$$

In summary, applying the law in equation (11), it is possible to change the bending stiffness of the active unit in a manner expressed in equation (9). One can increase the bending stiffness by using a positive $\alpha$ or decrease it by using a negative $\alpha$ compared with that of the host beam. 


\section{Control effects and stability issues}

In this section, the control effects and stability issues of the active unit are discussed based on theoretical and numerical studies on a clamped-free unit (namely, a cantilever). In the simulations, the host beam is made of aluminum and the piezoelectric patches are made of PIC 151. Parameters of these materials are listed in Appendix A. The geometrical parameters of the active unit are given in table 1.

Table 1: Geometrical parameters of the clamped-free active unit.

\begin{tabular}{lcll}
\hline & Length & \multicolumn{1}{c}{ Width } & Height \\
\hline Host beam & $l=0.05 \mathrm{~m}$ & $b=0.05 \mathrm{~m}$ & $h_{b}=0.005 \mathrm{~m}$ \\
Actuator and sensor & $l=0.05 \mathrm{~m}$ & $\begin{array}{l}b_{a}=0.044 \mathrm{~m}, \\
b_{s}=0.0025 \mathrm{~m}\end{array}$ & $h_{p}=0.0005 \mathrm{~m}$ \\
\hline
\end{tabular}

The first bending mode of the clamped-free unit is studied to reveal the control performances. Assume that the damping is low therefore can be ignored, then the resonant frequency of the first bending mode of the clamped-free unit is analytically obtained

$$
f_{n 1}=\frac{3.516}{l^{2}} \sqrt{\frac{D_{b}(\alpha)}{\rho_{b} b h_{b}+2 \rho_{p} b h_{p}}},
$$

in which, $\rho_{b}$ and $\rho_{p}$ are the density of the host beam and that of the piezoelectric patches, respectively. From equation (12) it can be seen that the bending stiffness $D_{b}$ is positively correlated to the resonant frequency, therefore the latter can be used as an indicator of the former.

The first bending mode of the clamped-free unit is also numerically studied using the finite element method (FEM). The simulations are done in the COMSOL Multiphysics software. 3D quadratic Lagrange elements are used in the FEM model, as shown in figure 2. The control law in equation (11) is applied on the actuators as electric boundary condition. Figure 3 shows the variation of the resonant frequency of the first bending mode when $\alpha$ changes. Both the theoretical and numerical results are illustrated in the figure. Figure 3 also shows the ratios between controlled and measured voltages $\left(V_{a} / V_{s}\right)$ for different $\alpha$ obtained using equation (11). From the numerical results, it can be seen that the applied control strategy is able to reduce the bending stiffness to be close to zero or increase it to some extent compared with that of the host beam. The theoretical and numerical results match well with each other when $\alpha$ satisfies $-0.7 \leq \alpha \leq 2.6$, the relative difference between them is less than $10 \%$. Obvious discrepancies between the theoretical and numerical results are observed when $\alpha<-0.7$ or $\alpha>2.6$. This difference is mainly caused by the actuators. The actuators are designed to bend the unit along the length direction ( $x$ axis). However, an unwanted bending along the width direction ( $y$ axis) is also caused since the patches are transversely isotropic. The importance of this unwanted bending increases as the absolute value of the ratio $V_{a} / V_{s}$ increases. Consequently, when $\alpha<-0.7$ or $\alpha>2.6$, the unwanted bending becomes non-negligible (for instance, see the mode shape at $\alpha=3$ in figure 3 ), the beam theory no longer holds very well in the unit's behaviors. 


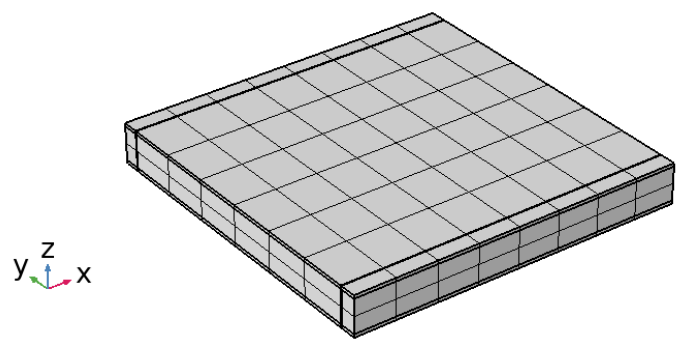

Figure 2: Mesh of the FE model.

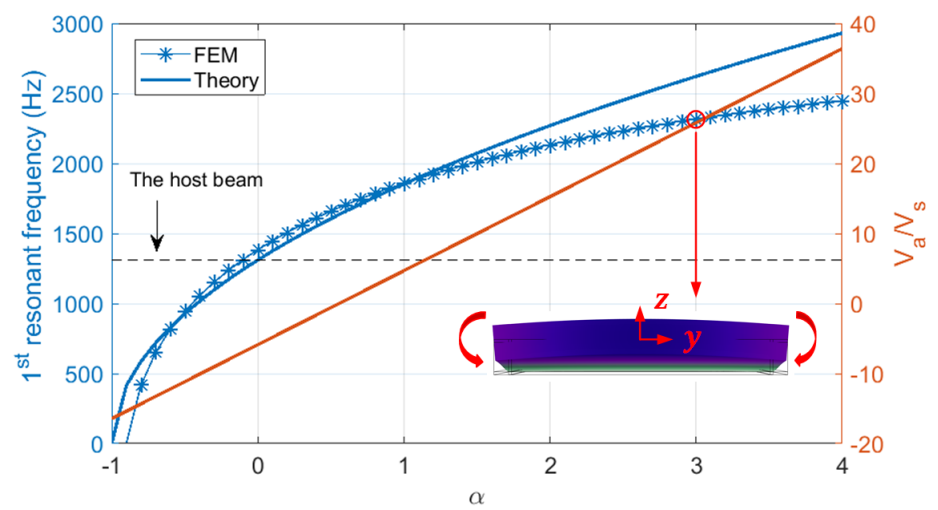

Figure 3: Theoretical and numerical resonant frequencies of the first bending mode as well as the ratios $V_{a} / V_{s}$ obtained using equation (11) for different $\alpha$ values. The mode shape at $\alpha=3$ obtained using the FEM is also shown in the figure in $Y Z$ view. The horizontal dashed line indicates the resonant frequency of the host beam.

With respect to the stability, according to equation (9), it can be seen that a positive $\alpha$ will cause no stability problem since the bending stiffness is increased. On the contrary, a negative $\alpha$ decreases the bending stiffness, more larger the absolute value of the negative $\alpha$ is, more the stiffness is reduced. Therefore, after a certain critical value the static bending stiffness becomes negative, the system is unstable. Theoretically, the active unit becomes unstable when $\alpha \leq-1$. However, as revealed in figure 3, the active unit behaves differently from the theoretical prediction when $\alpha$ is close to -1 , which means that the critical point of the stable zone could differ from the theoretical one. Therefore, for systems composed of the designed active units, if negative $\alpha$ values are used in the control law, the stability issue must be checked first.

The stability can be checked by only studying the pole related to the first resonant mode (the term "resonant" indicates that all rigid body modes are excluded since they are not controlled). According to the control theory, a linear system is stable when no pole of it is located in the right half part of the complex plane in the Laplace domain [38]. There are usually thousands of poles for a system. However, for the active systems presented here, there is no need to study all the poles, because as the bending stiffness decreases, the first resonant mode becomes unstable before others since it has the minimum resonant frequency. Therefore, only the pole related to the first resonant mode needs to be considered. For example, figure 4 shows the variation of the pole related to the first 


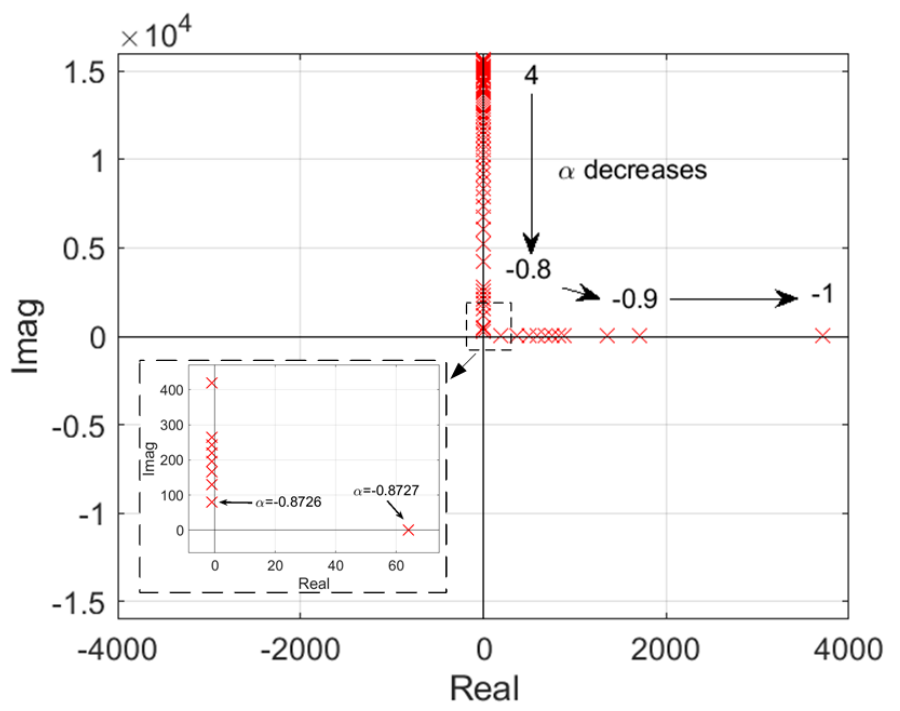

Figure 4: Variation of the pole related to the first mode of the clamped-free active unit when $\alpha$ decreases from 4 to -1 obtained using the FEM. The crosses represent the poles for different $\alpha$ values.

mode of the clamped-free active unit when $\alpha$ decreases from 4 to -1 . These results are obtained using the FEM. It can be seen that the clamped-free unit becomes unstable when $\alpha<-0.8726$, before the theoretical critical point.

\section{Applications}

\subsection{Spatial periodic waveguide with tunable band gaps}

First, the proposed active units are used to form a 1D spatial periodic waveguide. Band gaps in periodic structures are useful for vibration and noise control. Realizing band gaps nowadays is not a big challenge, however wider and even tunable band gaps in real time are still not easy to be obtained. It will be demonstrated that periodic waveguides composed of the proposed active units can have broad and controllable gaps.

Figure 5 shows the designed spatial periodic waveguide. It is obtained by alternating active units with passive beams. The waveguide can be divided into 20 identical cells. A zoom in on one of these cells is shown in figure 5. It should be clarified that, the term "active unit(s)" always only denotes the part composed of the patches and the host beam covered by the patches, as illustrated in figure 1, and the term "cell(s)" refers to the repetitive basic part composing a periodic waveguide. For instance, in figure 5, the cell contains an active unit and passive beams. The applied $\alpha$ values for all the active parts are the same. Geometrical parameters of the cells are listed in table 2.

The dispersion curves of flexural waves (A0 mode) corresponding to different $\alpha$ values are studied and the results are illustrated in figure 6 . In the figure, $\mathrm{k}$ represents the wavenumber and $l_{1}$ is the length of the cell. The simulations are done in COMSOL. Only a single cell illustrated in figure 5 is used in the simulation, Floquet periodic conditions are applied on the left and right boundaries of the cell to obtain the dispersion curves.

In figure $6(\mathrm{a})$ to (d), $\alpha$ equal to or smaller than 0 are used to soften the waveguide. Note that, the system is stable in all these studied cases. When $\alpha=0$ is used, the 


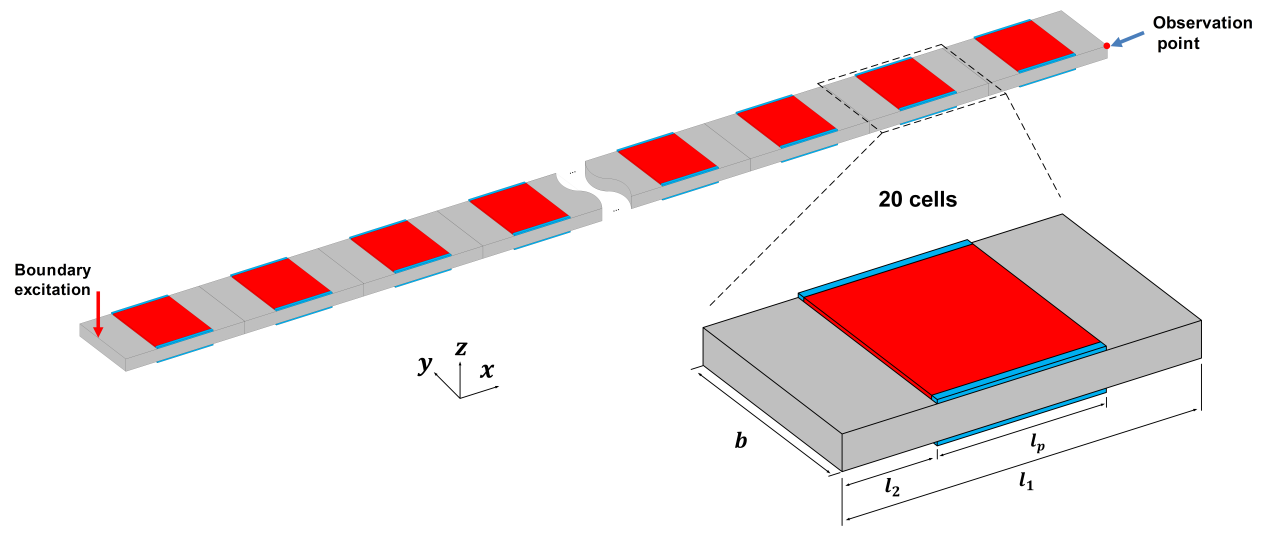

Figure 5: A spatial periodic waveguides containing 20 active units.

Table 2: Geometrical parameters for cells in the spatial periodic waveguide.

\begin{tabular}{lccc}
\hline & Length & Width & Height \\
\hline Host beam & $l_{1}=0.08 \mathrm{~m}$, & $b=0.05 \mathrm{~m}$ & $h_{b}=0.005 \mathrm{~m}$ \\
& $l_{2}=0.02 \mathrm{~m}$ & & \\
Actuator and sensor & $l_{p}=0.04 \mathrm{~m}$ & $\begin{array}{l}b_{a}=0.044 \mathrm{~m}, \\
b_{s}=0.0025 \mathrm{~m}\end{array}$ & $h_{p}=0.0005 \mathrm{~m}$ \\
\hline
\end{tabular}

waveguide is very close to a homogeneous beam, therefore the band gaps in this case are quite narrow. As the absolute value of the negative $\alpha$ increases, the lower boundaries of the first and second band gaps decrease, the upper boundaries remain nearly unchanged. Consequently, the widths of the gaps are significantly broadened. In figure 6(e) to (h), $\alpha$ with values larger than 0 are used to stiffen the waveguide. In these cases, as the $\alpha$ increases, the upper boundaries of the gaps move to higher frequencies and the lower boundaries remain almost at the original location, leading to wider gaps.

Figure 7 more clearly illustrates how the applied $\alpha$ value will influence the widths of the first and second band gaps. In the figure are also shown the ratios of the wavelengths at the upper frequencies of the second band gaps to the length of the patch. It can be seen that the control law works well until the wavelength is close to 1.7 times of the patch's length. Below this ratio, increasing $\alpha$ leads to less enlargement of the second band gap.

The vibrational properties of the finite waveguide are studied using the FEM in frequency domain. A transverse harmonic force is applied on the left end of the whole waveguide, and the displacement of a corner on the right end is studied (see figure 5). The structural loss factor for the aluminum and patches is set as $1 \times 10^{-4}$. Figure 8 shows the frequency response curves of the displacement at the studied location when different $\alpha$ values are applied. The frequency bands where the vibration level is low correspond to the band gaps. A solo peak inside a band gap observed in some cases are caused by localized modes [23]. One can see that results in figure 8 further verify the tunable band gaps of the proposed active waveguide. One can use a negative $\alpha$ to broaden the gaps and make them cover low frequency ranges. This feature could be very useful for low frequency vibration and noise control, which is still a challenge in many situations. One 


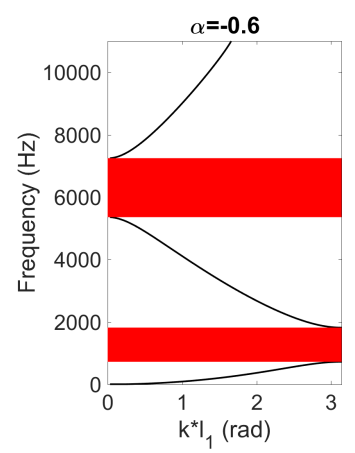

(a)

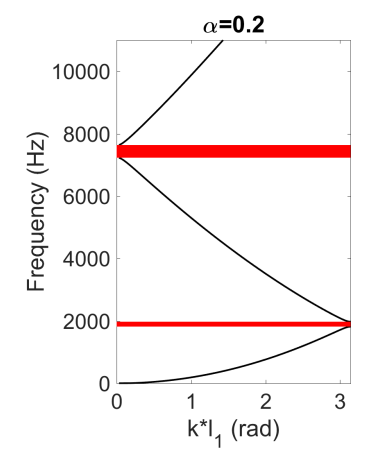

(e)

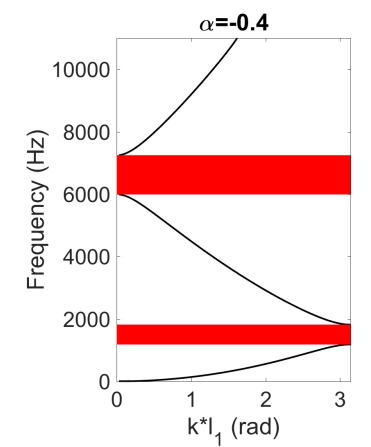

(b)

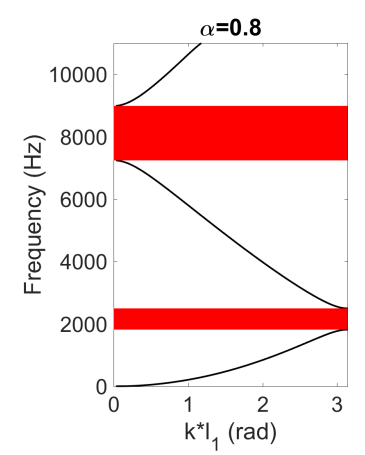

(f)

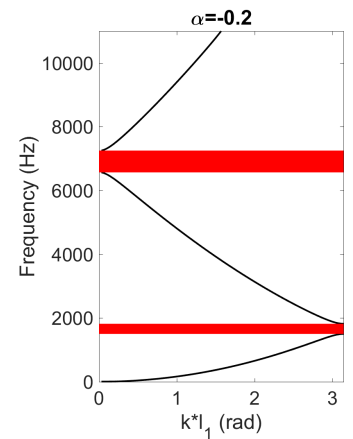

(c)

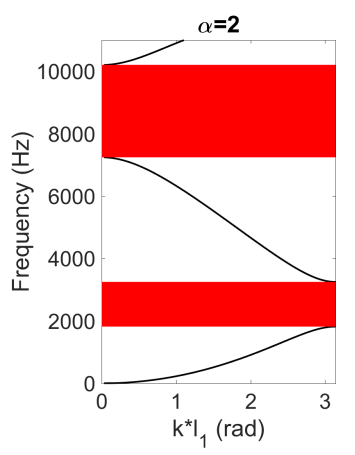

$(\mathrm{g})$

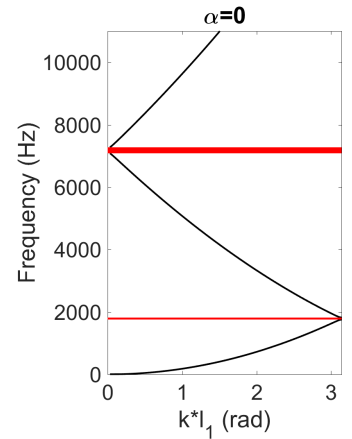

(d)

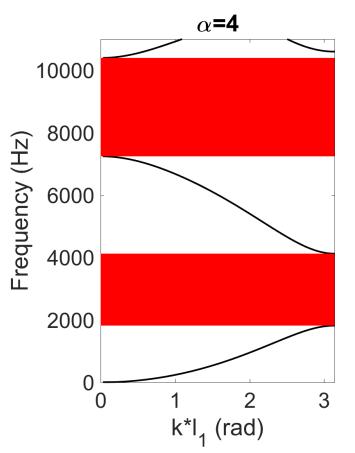

(h)

Figure 6: The dispersion curves of flexural waves (A0 mode) in the active waveguide corresponding to different $\alpha$ values. Color blocks indicate the band gaps. $\mathrm{k}$ represents the wavenumber and $l_{1}$ is the length of the cell. In (a) to (d) $\alpha$ values smaller than or equal to 0 are used to soften the waveguide; in (e) to (h) $\alpha$ values larger than 0 are used to stiffen the waveguide.

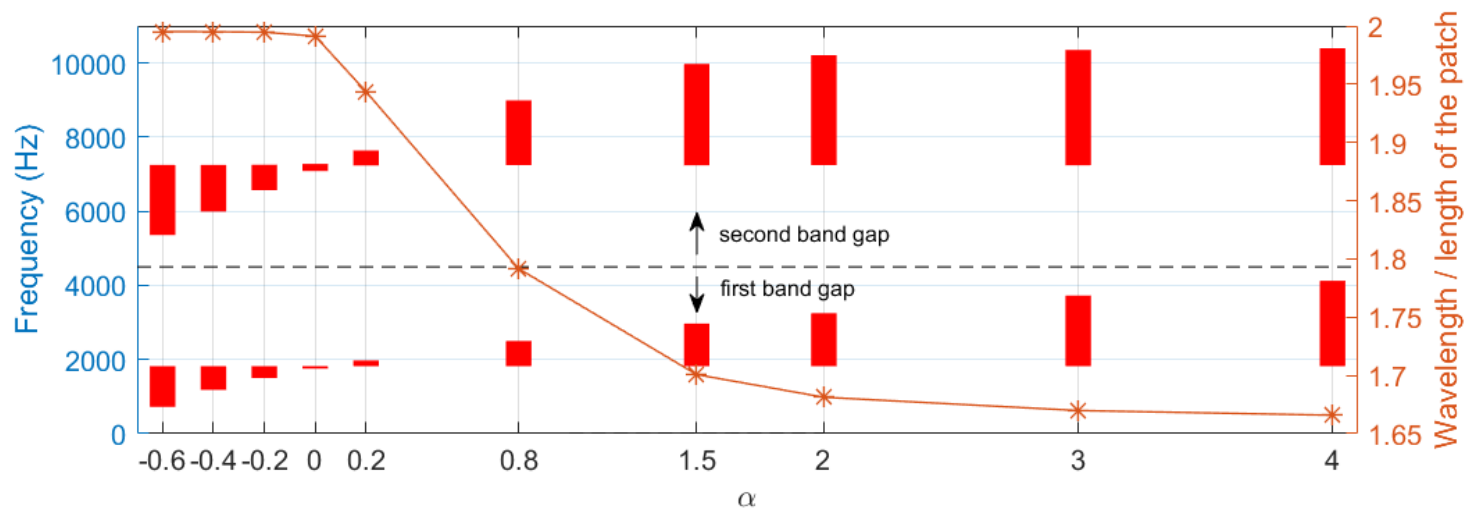

Figure 7: Left $\mathrm{Y}$ axis: widths of band gaps versus $\alpha$. Right $\mathrm{Y}$ axis: ratios of the wavelengths at the upper frequencies of the second band gaps to the length of the patch. 
can also use a positive $\alpha$ to make the gaps cover wider and higher frequency ranges for the purpose of vibration and noise reduction at interested frequencies.

\subsection{Spatiotemporal periodic waveguide for non-reciprocal wave propagation}

In this section the active units are used to form a spatiotemporal periodic waveguide to realize non-reciprocal wave propagation. Spatiotemporal periodic waveguides possess properties being modulated in both space and time. The non-reciprocal wave propagation effects inside them have been studied by many authors, as introduced in section 1 . However, very few propositions can be found on realization of such structures especially for guided elastic waves.

The designed waveguide has 20 cells with identical geometrical parameters, as shown in figure 9. Each cell contains 5 of these active units proposed in figure 1 . Therefore, in total there are 100 active units. The reason to design such a compact cell is motivated by the thought that in practice such cell may be realized by using only one complete patch with divided electrode segments on each surface of the host beam. The designed geometrical parameters of the cells are illustrated in table 3 .

Table 3: Geometrical parameters for cells in the spatiotemporal periodic waveguide.

\begin{tabular}{llll}
\hline & Length & Width & Height \\
\hline Host beam & $l_{1}=0.042 \mathrm{~m}$, & $b=0.01 \mathrm{~m}$ & $h_{b}=0.005 \mathrm{~m}$ \\
& $l_{2}=0.0005 \mathrm{~m}$ & & \\
Actuator and sensor & $l_{p}=0.008 \mathrm{~m}$ & $\begin{array}{l}b_{a}=0.008 \mathrm{~m}, \\
b_{s}=0.0005 \mathrm{~m}\end{array}$ & $h_{p}=0.0005 \mathrm{~m}$ \\
\hline
\end{tabular}

The local bending stiffnesses of the waveguide are modulated by changing the $\alpha$ values applied on the active units according to

$$
\alpha\left(x_{i}, t\right)=\alpha_{1}+\left(\alpha_{2}-\alpha_{1}\right) H\left[\cos \left(2 \pi f_{m} t-k_{m} x_{i}\right)\right], i=1,2, \ldots, 100,
$$

in which, $x_{i}$ is the central coordinate of the $i t h$ active unit, $H(\cdot)$ represents the Heaviside function, $f_{m}$ and $k_{m}$ are the frequency and wavenumber of the modulation wave, respectively. The wavenumber $k_{m}$ is determined by the wavelength $\lambda_{m}$ according to $k_{m}=2 \pi / \lambda_{m}$. In the simulations, 10 active units per wavelength are used to realize the modulation wave in a piecewise form. Therefore, the wavelength $\lambda_{m}$ is equal to two times of the cell's length, as illustrated in figure 9. The function in equation (13) approximates a rectangular wave as illustrated in figure 10, the $\alpha$ alternates between $\alpha_{1}$ and $\alpha_{2}$. Consequently, the bending stiffness of the $i t h$ active unit alternates between $K_{b}\left(\alpha_{1}\right)=\left(1+\alpha_{1}\right) Y_{b} b h_{b}^{3} / 12$ and $K_{b}\left(\alpha_{2}\right)=\left(1+\alpha_{2}\right) Y_{b} b h_{b}^{3} / 12$.

The transfer functions from left to right and from right to left of the waveguide are studied using the FEM. One pair of piezoelectric patches is placed on each side of the waveguide, as shown in figure 9 . To obtain the transfer function from left to right, the patches "L" are excited by a tone-burst voltage signal $v_{L}(t)$, the transient voltage responses $v_{R}(t)$ of the patches " $\mathrm{R}$ " are measured. The left to right transfer function is 


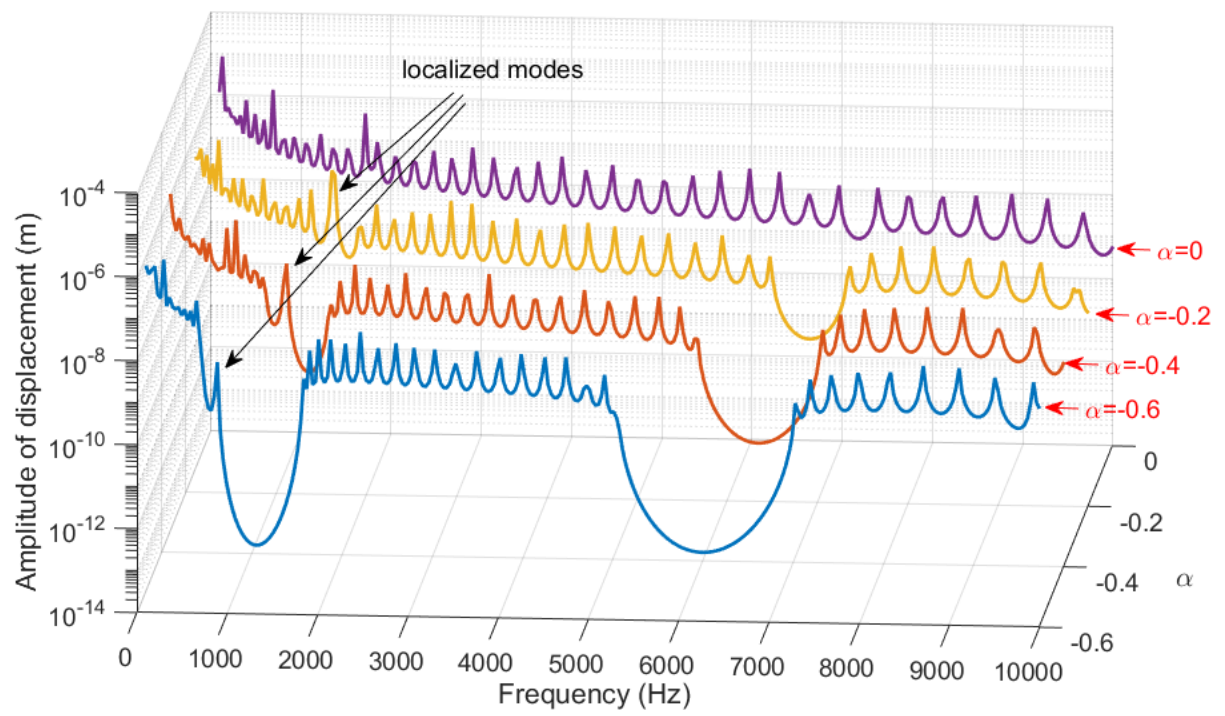

(a) Softening effects

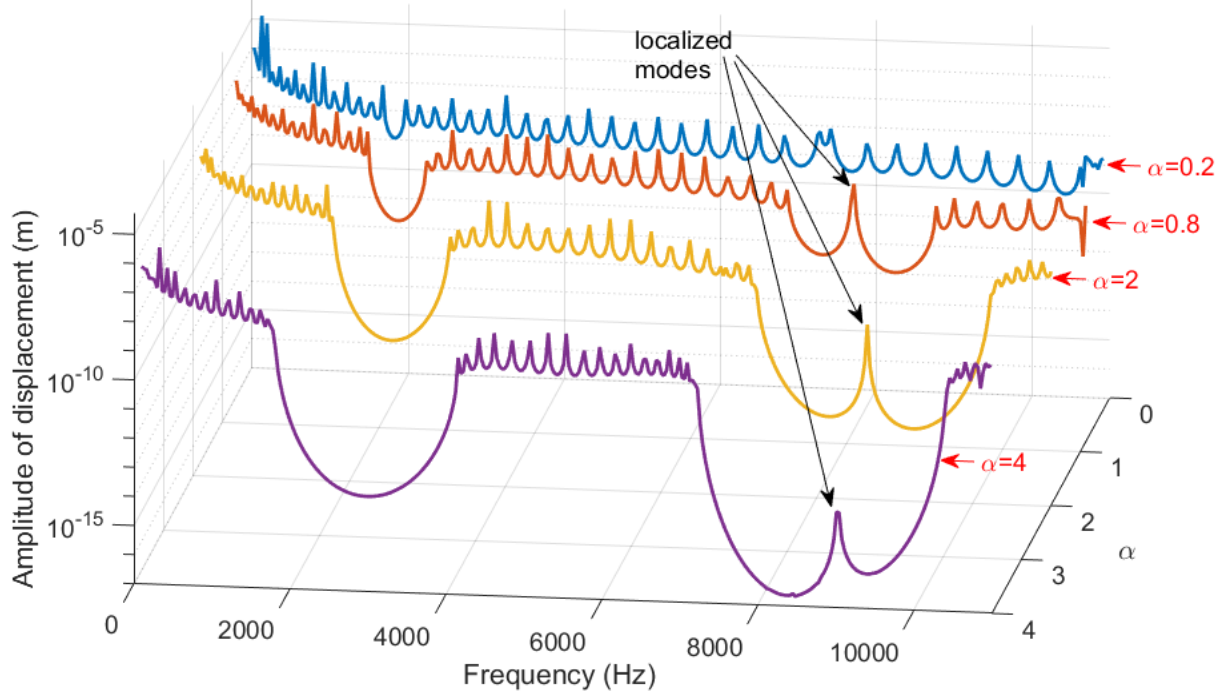

(b) Stiffening effects

Figure 8: Frequency response properties of the displacement at the studied location when different $\alpha$ values are used to (a) soften the structure or (b) stiffen the structure. 


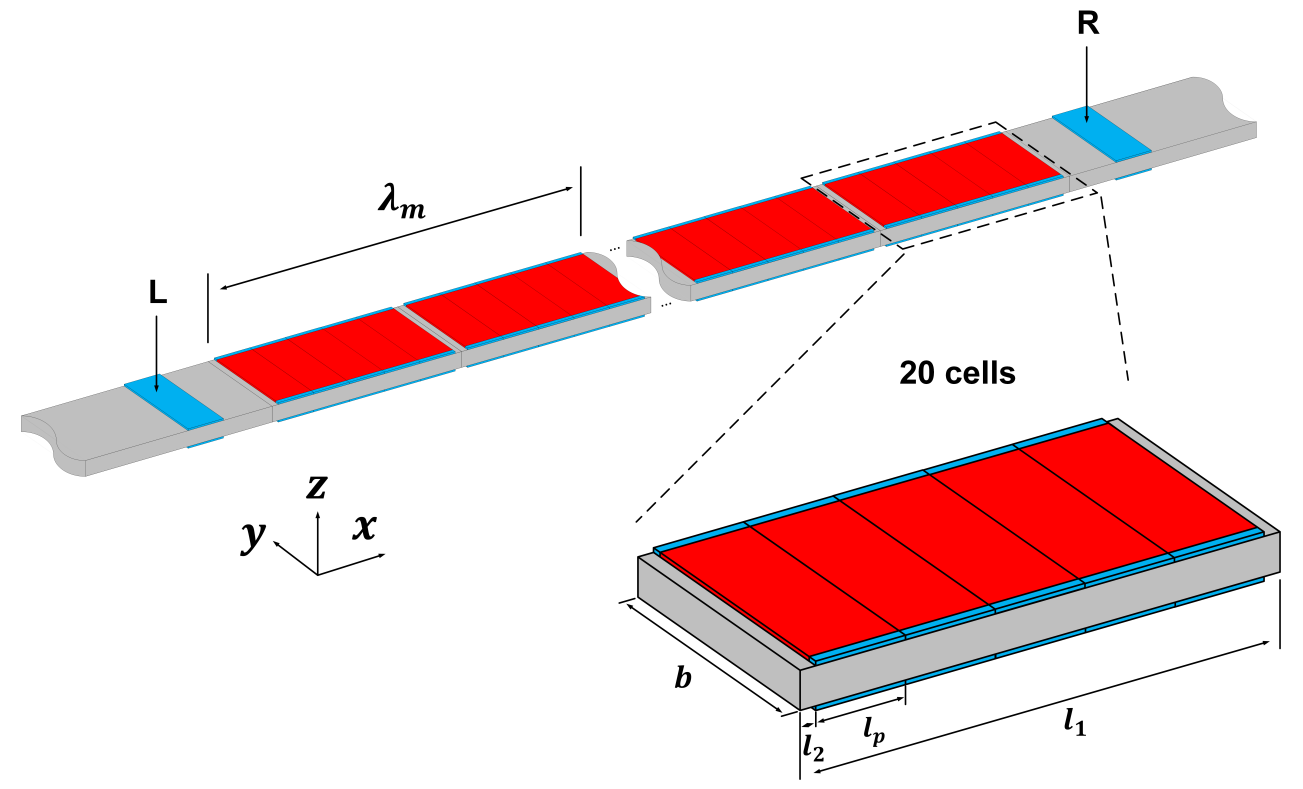

Figure 9: A spatiotemporal periodic waveguide containing 100 active units. A passive beam is connected to the active waveguide on each side, a pair of piezoelectric patches denoted by " $L$ " or " $R$ " is placed on each side of the waveguide.

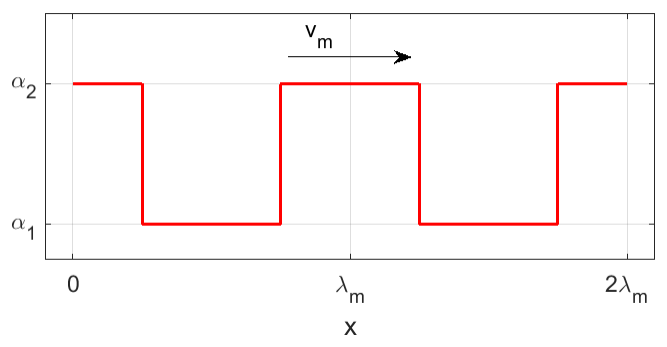

Figure 10: The applied modulation wave. $v_{m}=2 \pi f_{m} / k_{m}$ is the velocity and $\lambda_{m}$ represents the wavelength. 


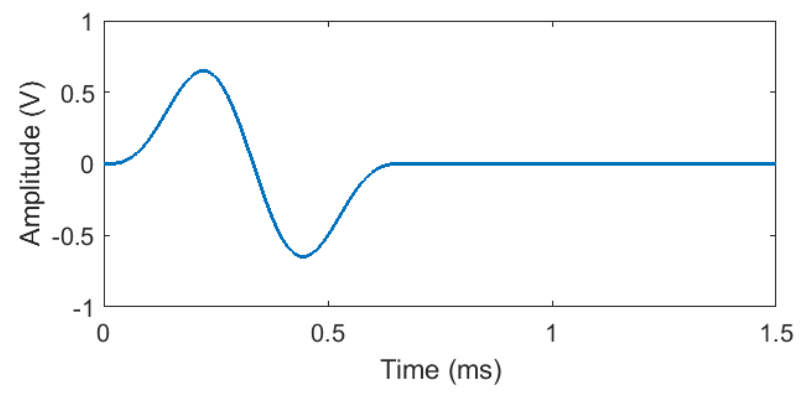

(a)

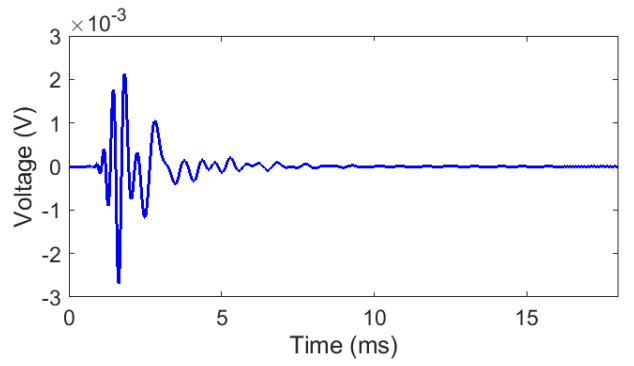

(b)

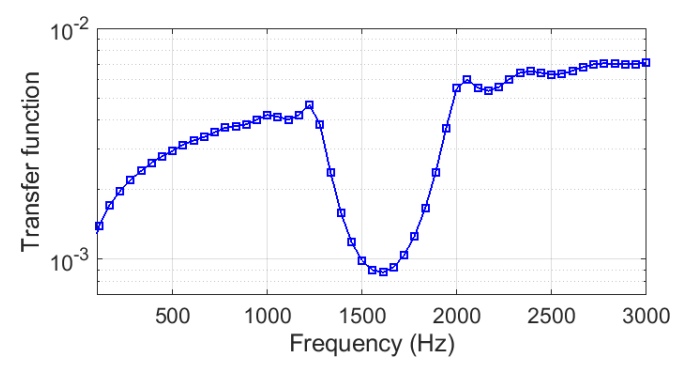

(c)

Figure 11: (a) The tone-burst voltage used for excitation, the central frequency is $1500 \mathrm{~Hz}$. (b) Transient voltage measured on the patches " $\mathrm{R}$ ". (c) Transfer function of the waveguide when $\alpha_{1}=0, \alpha_{2}=2$ and $f_{m}=0$.

therefore obtained using $F F T\left(v_{R}(t)\right) / F F T\left(v_{L}(t)\right)$, $F F T(\cdot)$ means Fast Fourier Transform. Similarly, the right to left transfer function is obtained by exciting the patches "R" and measure the response of patches "L". In the time domain simulations, the time step is $1 \times 10^{-5} \mathrm{~s}$, which is sufficient since as will be shown the interested frequencies are below $3000 \mathrm{~Hz}$. The damping is included by using the Rayleigh damping model, the coefficient for the mass matrix is 0.005 , and the one for the stiffness matrix is $3.18 \times 10^{-8}$.

As examples, $\alpha_{1}=0$ and $\alpha_{2}=2$ are used in the simulations for demonstration. First, the modulation frequency is set to be zero, namely $f_{m}=0$ in equation (13). In this case, the designed waveguide only has periodicity in space, the band gaps of it for left- and right-going waves are the same. To estimate the location of the first band gap, the one cycle tone-burst voltage with the central frequency equal to $1500 \mathrm{~Hz}$ shown in figure 11(a) is applied on the patches " $\mathrm{L}$ ". The voltage responses of the patches " $\mathrm{R}$ " are measured, as shown in figure 11(b). Note that, in the simulations the left and right passive beams are chosen to be long enough therefore the measured signals do not include the reflected waves from the two free ends of the whole waveguide. Using the measured voltage and the excitation voltage, the transfer function curve is obtained, as shown in figure 11(c). It can be observed that there is a band gap from around 1250 to $2000 \mathrm{~Hz}$.

When the modulation frequency is no longer zero, for example, if it is a positive value, the band gaps for left-going waves are moved to lower frequency bands, on the contrary, the gaps for right-going waves are moved to higher frequency bands. The shifted frequency value for the first gap of the flexural wave can be estimated using equation (14) when a harmonic modulation wave is used [31]. In our cases, a rectangular modulation wave is 
used. The major component of the Fourier series of a rectangular wave is the fundamental harmonic. Therefore, equation (14) could also be used in our cases to approximately estimate the shifted frequency bands.

$$
f_{\text {shift }}=\frac{f_{m}}{4}\left(\frac{\pi f_{m} \sqrt{3 \rho_{b}}}{k_{m}^{2} Y_{b} h_{b}}+2\right) \approx \frac{f_{m}}{2} .
$$

According to equation (14), to totally separate the left-going waves' band gap and the right-going waves' band gap, namely to obtain complete unidirectional gaps, the modulation frequency needs to be equal to or larger than the width of the gap. The width of the band gap of the waveguide with $\alpha_{1}=0$ and $\alpha_{2}=2$ is revealed in figure $11(\mathrm{c})$, which is $750 \mathrm{~Hz}$. Therefore, to have completely separated unidirectional band gaps, the modulation frequency must satisfy $f_{m} \geq 750 \mathrm{~Hz}$.

In figure 12, the left panel illustrates the corresponding left to right and right to left transfer functions when the modulation frequency is not zero, the right panel shows the measured voltage on patches "R" and "L" due to the excitation on patches "L" and "R". The transmission coefficients (namely the transfer functions here) are recommended tools to study the reciprocity [39, 40]. In figure $12(\mathrm{a})$, the modulation frequency is $400 \mathrm{~Hz}$. From the left panel it can be observed that for the left-going waves, a gap from 1444 to $2221 \mathrm{~Hz}$ is created; on the other hand, for the right-going waves, the gap is from 1055 to $1832 \mathrm{~Hz}$. Comparing these two gaps with the one obtained when $f_{m}=0$, it can be seen that the frequency shift caused by the moving modulation is close to the estimated value by using equation (14), which to some extend backs the accuracy of the simulations. The two gaps in figure 12(a) are not totally separated since the applied modulation frequency is smaller than the critical value. Therefore, in other simulations, the modulation frequency is chosen as $800 \mathrm{~Hz}$, larger than the critical value. In these cases, two totally separated unidirectional gaps are obtained, as shown in figure 12(b). Regarding the recorded voltages, from the right panel of figure 12 it can be seen that due to the non-reciprocity the recoded voltages on opposite sides are different.

Figure 13 shows the control signals of the 1st, 20th, 60th and 100th active units for $f_{m}=400 \mathrm{~Hz}$ and $f_{m}=800 \mathrm{~Hz}$. The excitation is applied on the patches "L". The 1 st active unit is the left-most one and the 100th is the right-most one in the waveguide. The control is local for each active unit, therefore the control acts only when the waves reach the corresponding active unit. The excitation voltage has a maximum amplitude equal to $0.65 \mathrm{~V}$, as shown in figure 11(a). Under this excitation, the control signal of the 1st active unit has the maximum amplitude among all the active units, around $0.06 \mathrm{~V}$ for both simulations. These results demonstrate that the required control voltages are totally within the reasonable range.

The duration of the incident wave is short in the simulations. Therefore, the phase difference between the incident wave and the modulation wave has some influences on the transmission properties of the waveguide. To study these influences, a initial phase $\varphi_{0}$ is introduced into the modulation wave, as shown in equation (15), while the initial phase of the incident wave keeps zero.

$$
\alpha\left(x_{i}, t\right)=\alpha_{1}+\left(\alpha_{2}-\alpha_{1}\right) H\left[\cos \left(2 \pi f_{m} t-k_{m} x_{i}+\varphi_{0}\right)\right], i=1,2, \ldots, 100
$$



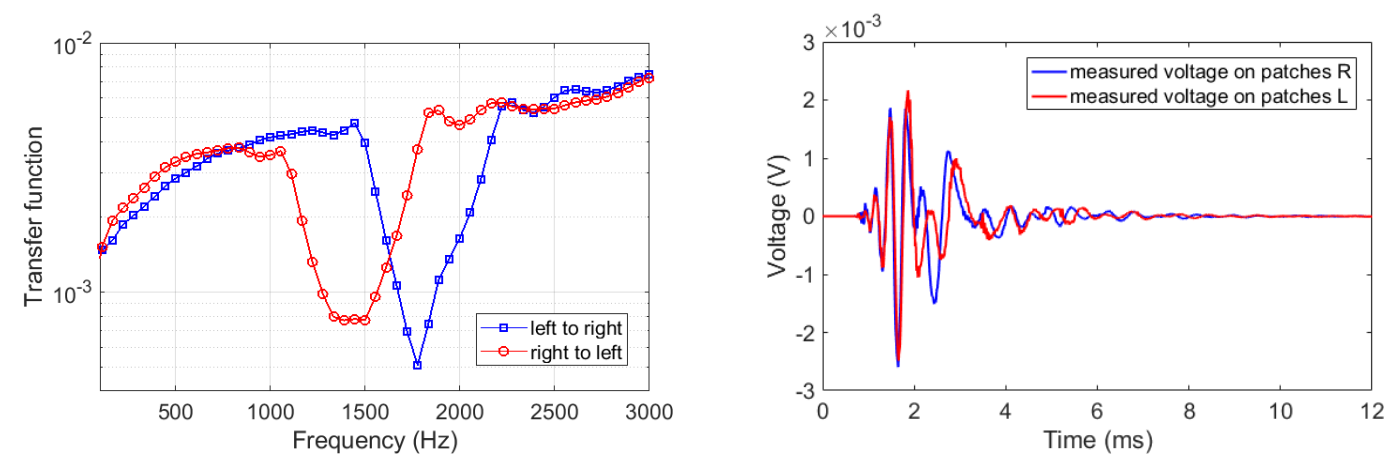

(a) $f_{m}=400 \mathrm{~Hz}$
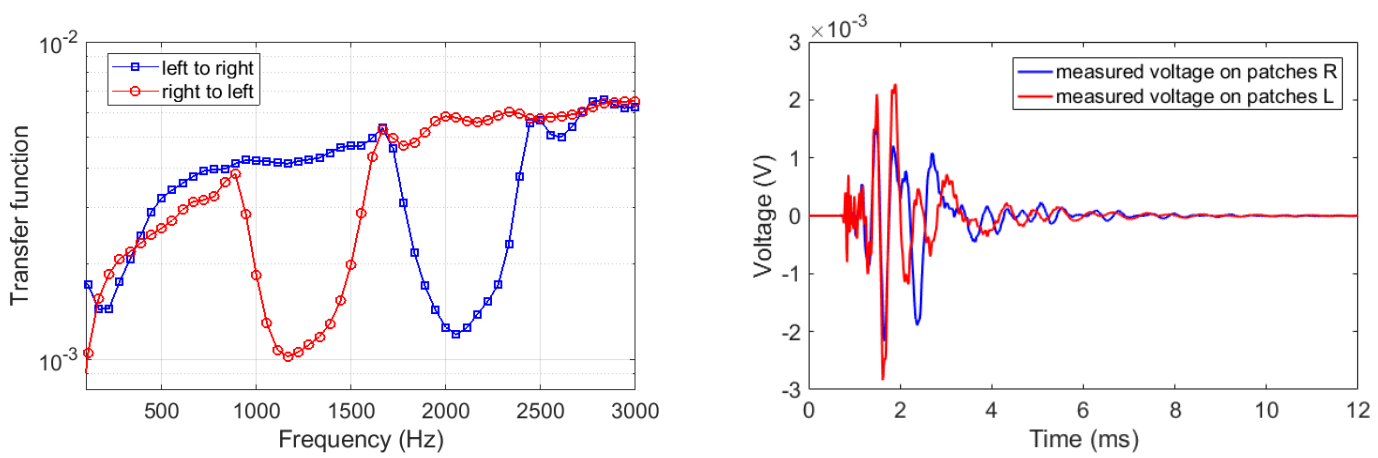

(b) $f_{m}=800 \mathrm{~Hz}$

Figure 12: Left panel: left to right and right to left transfer functions of the waveguide. Right panel: measured voltage on patches "R" and "L" due to the excitation on patches "L" and "R". The modulation parameters are $\alpha_{1}=0, \alpha_{2}=2$, and (a) $f_{m}=400 \mathrm{~Hz}$, (b) $f_{m}=800 \mathrm{~Hz}$.

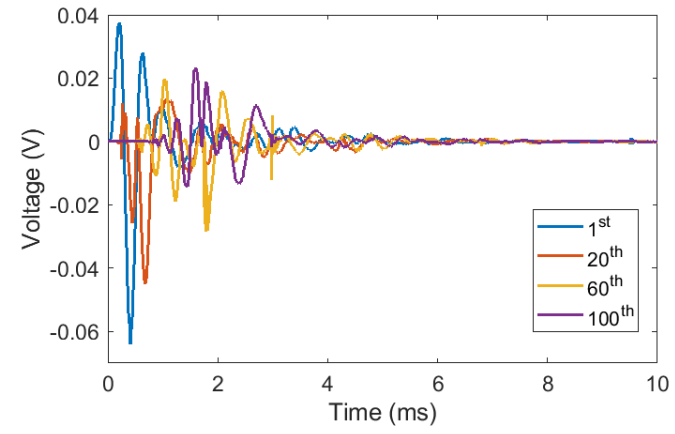

(a) $f_{m}=400 \mathrm{~Hz}$

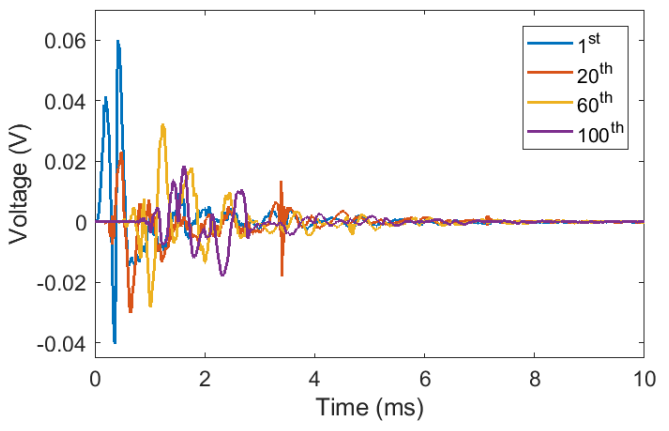

(b) $f_{m}=800 \mathrm{~Hz}$

Figure 13: Control signals for the 1st, 20th, 60th and 100th active units when $\alpha_{1}=0, \alpha_{2}=2$ and (a) $f_{m}=400 \mathrm{~Hz}$, (b) $f_{m}=800 \mathrm{~Hz}$. The excitation is on the left in both simulations. 


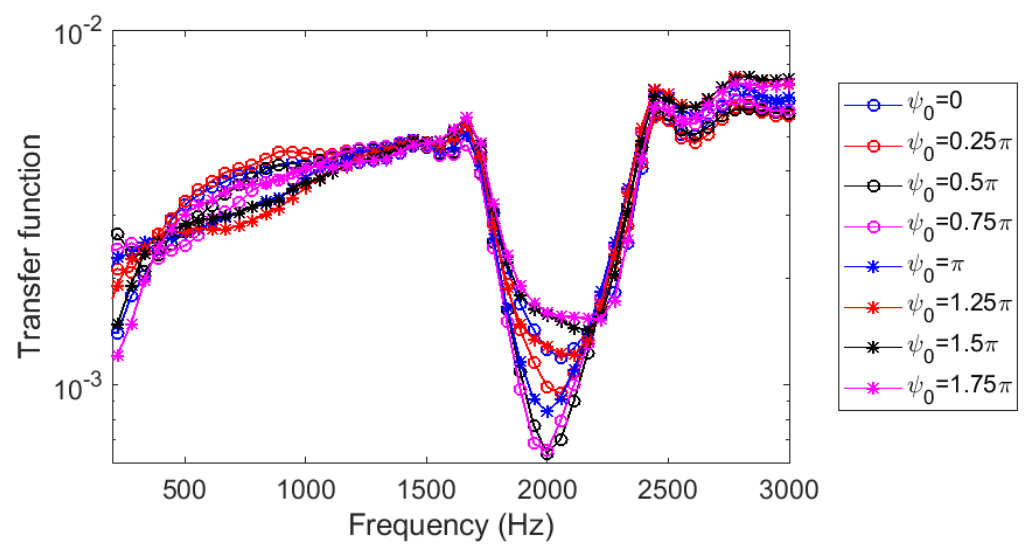

Figure 14: Influences of the initial phase $\varphi_{0}$ of the modulation wave on the transfer function. The excitation is applied on the left in each simulation.

8 different $\varphi_{0}$ values evenly chosen between 0 and $2 \pi$ have been studied, the corresponding transfer functions are illustrated in figure 14. The excitation is applied on the left in each simulation. It can be observed that the phase changes the depth of the gap. Increasing the cycle number of the incident wave can reduce the influences of phase, since it has been demonstrated that the phase has no influence on the transmission properties in frequency domain [33].

\section{Conclusions}

An active unit with controllable bending stiffness is proposed. The active unit is composed of a host beam and piezoelectric patches bonded on the beam surfaces. Some patches are used as sensors to measure the difference between the rotation angles at the two ends of the active unit. The other patches are used as actuators. A feedback control loop is used between the sensors and actuators. An appropriate control law is applied to control the bending stiffness. Due to the control, the bending stiffness is $(1+\alpha)$ times of that of the bare host beam. By choosing different $\alpha$ values, it is possible to obtain different stiffnesses for the active unit. A positive $\alpha$ stiffens the unit and a negative one softens it. Systems containing the designed active units are stable if $\alpha$ is larger than a certain critical value, which is negative and depends on the studied system. A simple method to check the stability is studying the pole related to the first resonant mode of the system. The pole must not be located in the right half part of the complex domain to guarantee a stable system.

The active units are included in a 1D spatial period waveguide to obtain tunable band gaps. Numerical results show that, by softening or stiffening the waveguide, the band gaps are broadened. Particularly, when the waveguide is softened, the first gap extends to low frequency ranges, which is very desired since controlling low frequency vibration and noise is not an easy task in many situations.

The active units are also used to realize a $1 \mathrm{D}$ spatiotemporal periodic waveguide for non-reciprocal wave propagation. The moving modulation of the local bending stiffnesses is realized by alternating the applied $\alpha$ for each active unit between two designed values 
according to a rectangular wave function. The non-reciprocal transmission through the waveguide is numerically verified. By choosing an appropriate modulation frequency, complete unidirectional band gaps are demonstrated. It is also demonstrated that the required control voltages are totally within a reasonable range.

\section{Acknowledgement}

This work was supported by the EUR EIPHI project (contract ANR-17-EURE-0002) and Bourgogne Franche-Comté region.

\section{Appendix A. Materials parameters}

The Young's modulus and density of aluminum are $70 \mathrm{GPa}$ and $2700 \mathrm{~kg} / \mathrm{m}^{3}$, respectively. The parameters of the PIC 151 are listed in table A.4. The in-plane Young's modulus of piezoelectric patches made by PIC 151 is $Y_{p}=1 / S_{11}^{E}$.

Table A.4: Material parameters of PIC 151.

\begin{tabular}{ccc}
\hline Symbol & Value & Property \\
\hline$S_{11}^{E}=S_{22}^{E}, S_{33}^{E}$ & $1.683 \times 10^{-11}, 1.9 \times 10^{-11}\left(\mathrm{~Pa}^{-1}\right)$ & Compliance matrix under \\
$S_{12}^{E}, S_{13}^{E}=S_{23}^{E}$ & $-5.656 \times 10^{-12},-7.107 \times 10^{-12}\left(\mathrm{~Pa}^{-1}\right)$ & constant electric field \\
$S_{44}^{E}=S_{55}^{E}, S_{66}^{E}$ & $5.096 \times 10^{-11}, 4.497 \times 10^{-11}\left(\mathrm{~Pa}^{-1}\right)$ & \\
\hline$d_{31}=d_{32}$ & $-2.14 \times 10^{-10}(\mathrm{C} / \mathrm{N})$ & Piezoelectric matrix \\
$d_{33}$ & $4.23 \times 10^{-10}(\mathrm{C} / \mathrm{N})$ & \\
$d_{24}=d_{15}$ & $6.1 \times 10^{-10}(\mathrm{C} / \mathrm{N})$ & Density \\
$\rho$ & $7760\left(\mathrm{~kg} / \mathrm{m}^{3}\right)$ & Dielectric permittivity \\
$\varepsilon_{1}^{\sigma}=\varepsilon_{2}^{\sigma}, \varepsilon_{3}^{\sigma}$ & $1936 \varepsilon_{0}, 2109 \varepsilon_{0}$ & under constant stress \\
\hline
\end{tabular}

\section{Appendix B. Relationship between the voltage on the sensors and the differ- ence of the rotation angles}

Constitutive equations of the sensors under open-circuited condition are

$$
\begin{gathered}
\epsilon_{x}^{p}=\frac{\sigma_{x}^{p}}{Y_{p}}+d_{31} E_{3}, \\
0=d_{31} \sigma_{x}^{p}+\varepsilon_{3}^{\sigma} E_{3} .
\end{gathered}
$$

According to equations (B.1), the strain can be expressed as

$$
\epsilon_{x}^{p}=-\frac{\varepsilon_{3}^{\sigma}-Y_{p} d_{31}^{2}}{d_{31} Y_{p}} E_{3} .
$$


Using the expression $\epsilon_{x}^{p}=-z \frac{\partial^{2} w}{\partial x^{2}}$ and equation (B.2), one can obtain

$$
z \frac{\partial^{2} w}{\partial x^{2}}=\frac{\varepsilon_{3}^{\sigma}-Y_{p} d_{31}^{2}}{d_{31} Y_{p}} E_{3} .
$$

Integrating the above equation along the thickness of the sensors leading to

$$
\frac{\partial^{2} w}{\partial x^{2}}=-\frac{2\left(\varepsilon_{3}^{\sigma}-Y_{p} d_{31}^{2}\right)}{h_{p}\left(h_{b}+h_{p}\right) d_{31} Y_{p}} V_{s} .
$$

in which, $V_{s}=-\int E_{3} d z$. Note that there are sensors on lower and upper surfaces, the integration must be performed from $\left(-h_{b} / 2-h_{p}\right)$ to $-h_{b} / 2$ and from $h_{b} / 2$ to $\left(h_{b} / 2+h_{p}\right)$.

Further integrating equation (B.4) from $x_{L}$ to $x_{R}$, one can have the final relationship between the sensed voltage and the difference of the rotation angles

$$
\theta_{\triangle}=\int_{x_{L}}^{x_{R}} \frac{\partial^{2} w}{\partial x^{2}} d x=-\frac{2 l\left(\varepsilon_{3}^{\sigma}-Y_{p} d_{31}^{2}\right)}{h_{p}\left(h_{b}+h_{p}\right) d_{31} Y_{p}} V_{s}
$$

\section{References}

[1] S A Cummer, J Christensen, and A Alù. Controlling sound with acoustic metamaterials. Nature Reviews Materials, 1(3):16001, 2016.

[2] M Rupin, F Lemoult, G Lerosey, and P Roux. Experimental demonstration of ordered and disordered multiresonant metamaterials for lamb waves. Physical review letters, 112(23):234301, 2014.

[3] Z Liu, X Zhang, Y Mao, Y Zhu, Z Yang, C Chan, and P Sheng. Locally resonant sonic materials. science, 289(5485):1734-1736, 2000.

[4] R Zhu, XN Liu, GK Hu, CT Sun, and GL Huang. A chiral elastic metamaterial beam for broadband vibration suppression. Journal of Sound and Vibration, 333(10):2759 $2773,2014$.

[5] R Zhu, XN Liu, GK Hu, CT Sun, and GL Huang. Negative refraction of elastic waves at the deep-subwavelength scale in a single-phase metamaterial. Nature communications, 5:5510, 2014.

[6] P Li, X Chen, X Zhou, G Hu, and P Xiang. Acoustic cloak constructed with thinplate metamaterials. International Journal of Smart and Nano Materials, 6(1):73-83, 2015.

[7] S H Mousavi, A B Khanikaev, and Z Wang. Topologically protected elastic waves in phononic metamaterials. Nature communications, 6:8682, 2015.

[8] M Collet, M Ouisse, and F Tateo. Adaptive metacomposites for vibroacoustic control applications. IEEE Sensors Journal, 14(7):2145-2152, 2014. 
[9] G Wang, J Cheng, J Chen, and Y He. Multi-resonant piezoelectric shunting induced by digital controllers for subwavelength elastic wave attenuation in smart metamaterial. Smart Materials and Structures, 26(2):025031, 2017.

[10] L Sirota, F Semperlotti, and A M Annaswamy. Tunable and reconfigurable mechanical transmission-line metamaterials via direct active feedback control. Mechanical Systems and Signal Processing, 123:117-130, 2019.

[11] L Airoldi and M Ruzzene. Design of tunable acoustic metamaterials through periodic arrays of resonant shunted piezos. New Journal of Physics, 13(11):113010, 2011.

[12] A Bergamini, T Delpero, L Simoni, L Lillo, M Ruzzene, and P Ermanni. Phononic crystal with adaptive connectivity. Advanced Materials, 26(9):1343-1347, 2014.

[13] F Tateo, M Collet, M Ouisse, MN Ichchou, KA Cunefare, and P Abbe. Experimental characterization of a bi-dimensional array of negative capacitance piezo-patches for vibroacoustic control. Journal of Intelligent Material Systems and Structures, 26(8):952-964, 2015.

[14] R Zhu, YY Chen, MV Barnhart, GK Hu, CT Sun, and GL Huang. Experimental study of an adaptive elastic metamaterial controlled by electric circuits. Applied Physics Letters, 108(1):011905, 2016.

[15] Y Chen, J Hu, and G Huang. A design of active elastic metamaterials for control of flexural waves using the transformation method. Journal of Intelligent Material Systems and Structures, 27(10):1337-1347, 2016.

[16] MH Ansari, MA Attarzadeh, M Nouh, and M Amin Karami. Application of magnetoelastic materials in spatiotemporally modulated phononic crystals for nonreciprocal wave propagation. Smart Materials and Structures, 27(1):015030, 2017.

[17] G Matten, M Collet, S Cogan, and E Sadoulet-Reboul. Synthetic impedance for adaptive piezoelectric metacomposite. Procedia Technology, 15:84-89, 2014.

[18] C Sugino, M Ruzzene, and A Erturk. Design and analysis of piezoelectric metamaterial beams with synthetic impedance shunt circuits. IEEE/ASME Transactions on Mechatronics, 23(5):2144-2155, 2018.

[19] M Collet, M Ouisse, and M Ichchou. Structural energy flow optimization through adaptive shunted piezoelectric metacomposites. Journal of Intelligent Material Systems and Structures, 23(15):1661-1677, 2012.

[20] Y Wang, F Li, and Y Wang. Active feedback control of elastic wave metamaterials. Journal of Intelligent Material Systems and Structures, 28(15):2110-2116, 2017.

[21] F Li, C Zhang, and C Liu. Active tuning of vibration and wave propagation in elastic beams with periodically placed piezoelectric actuator/sensor pairs. Journal of Sound and Vibration, 393:14-29, 2017. 
[22] YY Chen, GL Huang, and CT Sun. Band gap control in an active elastic metamaterial with negative capacitance piezoelectric shunting. Journal of Vibration and Acoustics, 136(6):061008, 2014.

[23] Y Fan, M Collet, M Ichchou, L Li, O Bareille, and Z Dimitrijevic. A wave-based design of semi-active piezoelectric composites for broadband vibration control. Smart Materials and Structures, 25(5):055032, 2016.

[24] X Li, YY Chen, GK Hu, and GL Huang. A self-adaptive metamaterial beam with digitally controlled resonators for subwavelength broadband flexural wave attenuation. Smart Materials and Structures, 27(4):045015, 2018.

[25] K Yi, M Collet, M Ichchou, and L Li. Flexural waves focusing through shunted piezoelectric patches. Smart Materials and Structures, 25(7):075007, 2016.

[26] P Celli, S Gonella, V Tajeddini, A Muliana, S Ahmed, and Z Ounaies. Wave control through soft microstructural curling: bandgap shifting, reconfigurable anisotropy and switchable chirality. Smart Materials and Structures, 26(3):035001, 2017.

[27] Y Chen, X Li, H Nassar, G Hu, and G Huang. A programmable metasurface for real time control of broadband elastic rays. Smart Materials and Structures, 27(11):115011, 2018.

[28] L Ning, YZ Wang, and YS Wang. Active control of elastic metamaterials consisting of symmetric double helmholtz resonator cavities. International Journal of Mechanical Sciences, 153-154:287 - 298, 2019.

[29] DW Wright and RSC Cobbold. Acoustic wave transmission in time-varying phononic crystals. Smart Materials and Structures, 18(1):015008, 2008.

[30] D Casagrande, P Gardonio, and M Zilletti. Smart panel with time-varying shunted piezoelectric patch absorbers for broadband vibration control. Journal of Sound and Vibration, 400:288-304, 2017.

[31] G Trainiti and M Ruzzene. Non-reciprocal elastic wave propagation in spatiotemporal periodic structures. New Journal of Physics, 18(8):083047, 2016.

[32] H Nassar, XC Xu, AN Norris, and GL Huang. Modulated phononic crystals: Nonreciprocal wave propagation and willis materials. Journal of the Mechanics and Physics of Solids, 101:10-29, 2017.

[33] K Yi, S Karkar, and M Collet. One-way energy insulation using time-space modulated structures. Journal of Sound and Vibration, 429:162-175, 2018.

[34] K Yi, M Collet, and S Karkar. Frequency conversion induced by time-space modulated media. Physical review B, 96(10):104110, 2017. 
[35] C Croënne, JO Vasseur, O Bou Matar, M-F Ponge, PA Deymier, A-C HladkyHennion, and B Dubus. Brillouin scattering-like effect and non-reciprocal propagation of elastic waves due to spatio-temporal modulation of electrical boundary conditions in piezoelectric media. Applied Physics Letters, 110(6):061901, 2017.

[36] F Li and C Zhang. Active localization of wave propagation in elastic beams using periodic placement of piezoelectric actuator/sensor pairs. Journal of Applied Physics, 124(8):085106, 2018.

[37] L Li, S Yin, X Liu, and J Li. Enhanced electromechanical coupling of piezoelectric system for multimodal vibration. Mechatronics, 31:205-214, 2015.

[38] G F Franklin, J D Powell, A Emami-Naeini, and J D Powell. Feedback control of dynamic systems, volume 3. Addison-Wesley Reading, MA, 1994.

[39] D Jalas, A Petrov, M Eich, W Freude, S Fan, Z Yu, R Baets, M Popovi, A Melloni, and J D. Joannopoulos. What is and what is not an optical isolator. Nature Photonics, 7(8):579-582, 2013.

[40] AA Maznev, AG Every, and OB Wright. Reciprocity in reflection and transmission: What is a phonon diode? Wave Motion, 50(4):776-784, 2013. 